\title{
Adverse events with fatal outcome associated with alemtuzumab treatment in multiple sclerosis
}

Trygve Holmøy ${ }^{1,2^{*}} \mathbb{D}$, Børre Fevang ${ }^{3,4}$, David Benee Olsen ${ }^{5}$, Olav Spigset ${ }^{6,7}$ and Lars B $\varnothing^{8,9}$

\begin{abstract}
Objective: Sporadic fatal adverse events have been reported during treatment of multiple sclerosis with alemtuzumab. To provide a systematic overview, we searched the centralized European Medicines Agency database of suspected adverse reactions related to medicinal products (EudraVigilance) for fatal adverse events associated with treatment with alemtuzumab (Lemtrada ${ }^{\circledR}$ ) for multiple sclerosis. Four independent reviewers with expertise on MS, clinical immunology, infectious diseases and clinical pharmacology reviewed the reports, and scored the likelihood for causality.

Results: We identified nine cases with a probable and one case with a possible causal relationship between alemtuzumab treatment and a fatal adverse event. Six of these patients died within one month after treatment; one from intracerebral hemorrhage, two from acute multiple organ failure and septic shock, one from listeriosis, one from pneumonia and one from agranulocytosis. Four patients died several months after administration of alemtuzumab from either autoimmune hepatitis, immune-mediated thrombocytopenia, autoimmune hemolytic anemia or agranulocytosis. Four of the 10 cases had been published previously in case reports or congress abstracts. Fatal adverse events related to treatment with alemtuzumab occur more frequently than previously published in the literature. A significant proportion occurs in the first month after treatment.
\end{abstract}

Keywords: Multiple sclerosis, Treatment, Alemtuzumab, Adverse event

\section{Introduction}

Alemtuzumab is a humanized monoclonal antibody directed against CD52, and is regarded as one of the most efficacious drugs for treatment of relapsing-remitting multiple sclerosis (MS) [1]. Alemtuzumab induces a profound decrease of $\mathrm{T}$ and $\mathrm{B}$ lymphocytes, with a gradual recovery starting one month after administration [2, 3]. Even though alemtuzumab is generally considered safe, serious adverse reactions have been identified, including infections, immune-mediated thrombocytopenia and thyroiditis [3]. Following regulatory approval of alemtuzumab for relapsing remitting MS in 2013 by the European Medicines Agency (EMA) and in 2014 by the U.S.

\footnotetext{
*Correspondence: trygve.holmoy@medisin.uio.no

${ }^{1}$ Department of Neurology, Akershus University Hospital, Post Office Box 1000, 1478 Lørenskog, Norway

Full list of author information is available at the end of the article
}

Food and Drug Administration (FDA), there have been reports of severe and even fatal suspected adverse effects. These include listeriosis [4, 5], alveolar hemorrhage [6], neutropenia with staphylococcus infection [7], autoimmune hemolytic anemia with necrotizing leukoencephalopathy [8], and hemophagocytic lymphohistiocytosis [9].

These concerns led us to perform a systematic search for information on fatal cases following treatment with alemtuzumab in MS, retrieving data from the European database of suspected adverse drug reaction reports (EudraVigilance).

\section{Main text \\ Methods}

On November 19, 2018 we searched EudraVigilance for reports with product name "Lemtrada" as the suspect (or interacting) drug, and with the Medical Dictionary for Regulatory Activities (MedDRA) indication high-level 
term "Multiple sclerosis, acute and progressive", using the EudraVigilance Data Analysis System (EVDAS). Only reactions classified as "fatal" were included, as well as cases with the reaction outcome "fatal" and with Reaction Seriousness Death set to "Yes". The search included post-marketing spontaneous reports and reports from clinical studies from the European Economic Area, i.e. the European Union, Iceland, Liechtenstein and Norway.

Four reviewers with clinical and research experience in MS and neuroimmunology (LB and TH), clinical immunology and infectious diseases (BF) and clinical pharmacology (OS), independently reviewed the full Council for International Organization of Medical Sciences (CIOMS) reports and case narratives reports and scored the likelihood for causality in one of the four groups $>85 \%, 85-50 \%$, $50-35 \%$ or $<15 \%$. The cases were then discussed and the fatal adverse event assessed as related or unrelated to alemtuzumab, using guidance from the FDA and the World Health Organization and Uppsala Monitoring Centre $[10,11]$. Based on the known safety profile and biological effects of alemtuzumab, we considered immunosuppression, infection or hyperinflammation in close proximity of treatment, as well as secondary autoimmunity occurring months after treatment, as plausible consequences of alemtuzumab. As previously described for the assessment of the association between acute acalculous cholecystitis and alemtuzumab [12], the related cases were further subdivided as either probable or possible from the plausibility and robustness of the evidence, including whether alternative explanations could be reasonably ruled out from the available data. Case reports lacking information essential for the assessment of causality, including the temporal relationship between alemtuzumab treatment and the adverse event, disease history or concomitant medication, or where duplication could not be excluded, were discarded.

\section{Results}

After exclusion of duplicates, including two cases of fatal autoimmune hepatitis occurring almost simultaneously in the same country which were not marked as duplications in the case reports, there were 17 unique cases. In 10 of these (nine female and one male) the fatal adverse events were considered to be related to alemtuzumab. Clinical characteristics of these cases are shown in Table 1. All these patients were adults. The age was not further specified for one case, the others ranged from 34 to 47 years.

In nine unique cases (No. 1-9 in Table 1) all reviewers considered that the fatal adverse event was probably to be caused by alemtuzumab. Six of the patients (No. 1-6) died within one month after alemtuzumab infusion. All patients who died within one month had only received one alemtuzumab cycle. Five of these patients (No. 2-6) died from infection or multiple organ failure and septic shock, whereas the sixth (No. 1) developed hypertension and a cytokine storm and died from an intracerebral hemorrhage five days after receiving the first alemtuzumab infusion. Autopsy revealed necrotizing vasculopathy, but did not confirm that the patient had MS. The reporting physician concluded that causality with alemtuzumab was unlikely, as cerebral hemorrhage was not a known adverse event of alemtuzumab. The patient had also received the antithrombotic drug certoparin sodium. Given the immediate temporal relationship and the recent report of early strokes associated with alemtuzumab treatment from the FDA [13], we concluded that causality was probable. Notably, increasing blood pressure, which was reported in this patient, was recently suggested to be a characteristic feature of alemtuzumab-induced stroke [14].

The remaining three patients in whom a causal role of alemtuzumab were considered probable (No. 7-9) were all female, and died from secondary autoimmunity 8 to 18 months after the last alemtuzumab infusion. Of these, one patient died from immune-mediated thrombocytopenia and brain stem hemorrhage, one from autoimmune hepatitis, and one from autoimmune hemolytic anemia, disseminated intravascular coagulation and septic shock. Immune-mediated thrombocytopenia was diagnosed several weeks prior to the intracranial hemorrhage, but did not respond to treatment with corticosteroids and intravenous immunoglobulins.

In five cases the fatal adverse event [suicide $(n=2)$, cancer $(n=2)$ and status epilepticus $(n=1)]$ were considered unlikely to be related to alemtuzumab. In addition, two cases were considered unclassifiable. These were neonates weighing less than $500 \mathrm{~g}$, who both died within one day after induced labor. The mother had been treated with alemtuzumab during pregnancy.

The reviewers disagreed substantially on two cases. One patient (No. 10) developed agranulocytosis 17 months after the second course of alemtuzumab, followed by colitis, aspergillus pneumonia and death from multiple organ failure. $\mathrm{CD} 4^{+} \mathrm{T}$ cells were also low prior to death. Two reviewers scored the likelihood of a causal relationship as $<50 \%$. It was, however, agreed to emphasize that neutropenia grade III or IV have been reported in $1.5 \%$ of MS patients in the second year after alemtuzumab treatment [15], and that it was plausible that neutropenia in combination with low $\mathrm{CD} 4^{+} \mathrm{T}$ cell counts induced by alemtuzumab contributed to aspergillus pneumonia and death. A causal relationship with alemtuzumab was therefore considered possible. The second patient, who had aggressive MS, developed status epilepticus few days after alemtuzumab, followed by aspiration pneumonia, sepsis and colon bleeding, and died after 40 days. In spite of a close temporal relationship with alemtuzumab most reviewers considered that status 


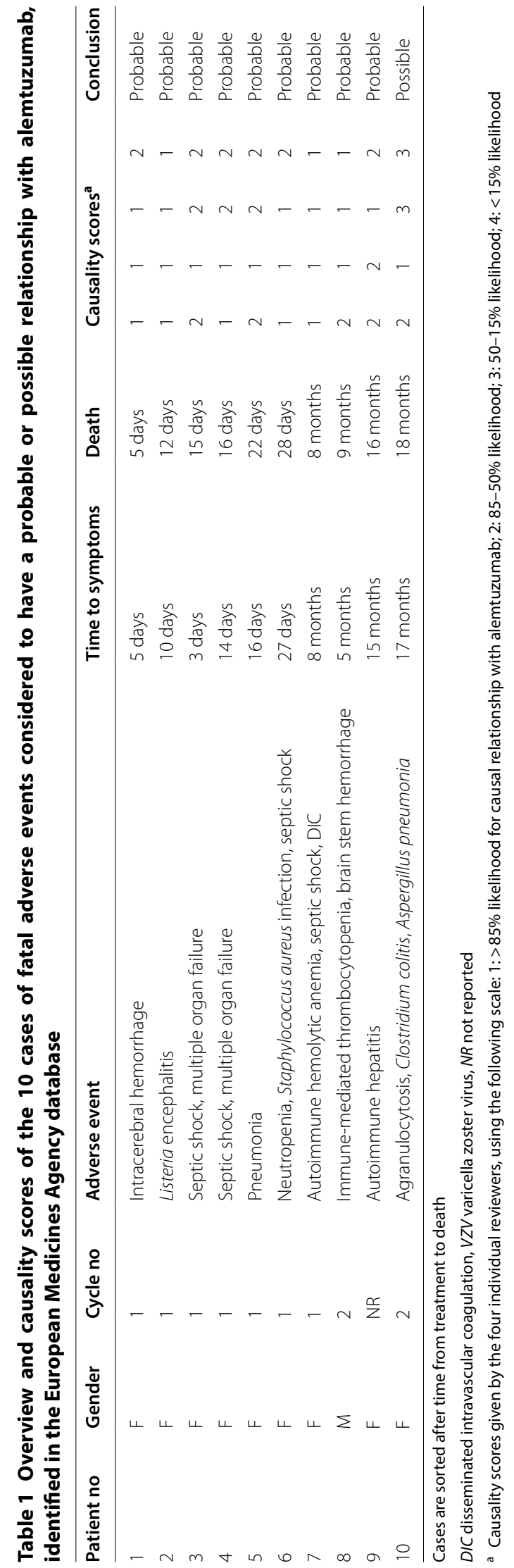


epilepticus was more likely caused by aggressive MS, and that causality therefore was unlikely.

\section{Discussion}

We identified 9 case reports of MS patients with fatal adverse events considered to be probably caused by alemtuzumab, and one with a possible causal relationship. Four of these cases (No. 2 and 6-8) have previously been published or reported on meetings $[4,7,16,17]$, whereas six have not been published. Our results were shared with the Pharmacovigilance Risk Assessment Committee in EMA before EMA initiated an article 20 review of Lemtrada on April 122019.

As expected from the pharmacodynamic properties and known adverse drug reaction profile of alemtuzumab, fatal adverse events either occurred early and were characterized by immunosuppression, hyperinflammation or stroke, or occurred several months later and were characterized by secondary autoimmunity. Secondary autoimmunity is known to occur frequently after alemtuzumab treatment in MS patients, and is suggested to be mediated by the reappearance of naïve immunologically active $B$ cells while regulatory $\mathrm{T}$ cells are still suppressed [18]. All cases of fatal secondary autoimmunity occurred within a time frame that is compatible with such a hypothesis. It is also known that listeriosis occurs during the first weeks after treatment with alemtuzumab, possibly reflecting marked and transient acute effects on both the adaptive and innate immunity, including impaired function of remaining immune cells [19]. The underlying mechanisms of such acute reactions is believed to include a programmed release of cytokines from natural killer cells, triggered by Fc cross-linking [20].

After we performed our search, FDA issued a warning related to 13 cases of and hemorrhagic stroke or arterial dissection occurring shortly after the patients had received alemtuzumab, mostly within one day after infusion [13]. This, along with the recently published report of eight cases of acalculous cholecystitis in close time proximity to alemtuzumab treatment [12], support a hypothesis of hyperinflammation after alemtuzumab treatment despite routine prophylaxis with corticosteroids.

Immune-mediated thrombocytopenia occurs in approximately $2 \%$ of MS patients treated with alemtuzumab [21]. It usually responds well to standard treatment with corticosteroids, although the fatal case reported here indicates that this is not always the case. The finding of only one case of immune-mediated thrombocytopenia with fatal outcome may however suggest that obligate screening for thrombocytopenia clearly limits the consequences of this adverse event. The same may be the case for glomerulonephritis, which was not recorded in this dataset.

Underreporting of adverse events is frequent, and even for severe adverse events it is estimated that only $1-10 \%$ of adverse events are reported [22]. Although underreporting may be less frequent for fatal adverse events, a PubMed search identified two fatal cases in Europe that were not registered in EudraVigilance [8, 9]. As some cases are likely neither published nor reported, the number of fatal adverse events in Europe may exceed the 12 events identified by us.

Whereas patients are routinely screened for secondary autoimmunity every month for at least four years after receiving alemtuzumab, the first weeks after treatment have received relatively little attention. Our results indicate that life threatening adverse events could be more frequent during this period. It should, however, be taken into consideration that in general, associations between drug treatment and adverse events are more easily recognized, and therefore also more often reported, shortly after commencement of drug therapy. In our material, the initial events mostly included infections and hyperinflammation. Prophylactic treatment with antibiotics has been suggested in addition to the antiviral therapy that is generally used [23], and could perhaps prevent listeriosis more effectively than diet advice alone. Weekly hematological screening the first period following treatment could possibly limit the consequences of early agranulocytosis [7], and monitoring of blood pressure could possibly prevent early cerebral hemorrhages [14]. Early adverse effects due to hyperinflammation can, however, at present neither be predicted nor fully prevented.

\section{Conclusions}

Fatal adverse events related to treatment with alemtuzumab occur more frequently than previously published in the literature, and seem to be most common during the first month after treatment. Patients, physicians and regulatory authorities should be aware of the serious risks associated with alemtuzumab treatment, which must be weighed against the high and durable clinical efficacy.

\section{Limitations}

- We had not access to full medical records. Although the CIOMS reports were generally quite detailed, we may have missed relevant information.

- The number of multiple sclerosis patients treated with alemtuzumab is not in the public domain. We can therefore not calculate the frequency of fatal adverse events.

- We may have missed cases that have not been reported to EudraVigilance.

\section{Abbreviations}

CD: cluster of differentiation; CIOMS: Council for International Organizations in Medical Sciences; EVDAS: EudraVigilance Data Analysis System; EMA: European Medicines Agency; FDA: Federal Drug Agency; MS: multiple sclerosis. 


\section{Acknowledgements}

The authors acknowledge Ane Simensen at The Norwegian Medicines Agency for collecting data from EudraVigilance.

\section{Authors' contributions}

$\mathrm{TH}$ and DBO designed the study. TH, BF, OS and $L B$ analyzed the data. TH drafted the manuscript. All authors revised the manuscript for intellectual content. All authors read and approved the final manuscript.

\section{Funding}

No targeted funding was obtained for this study.

\section{Availability of data and materials}

The authors are not permitted to share the case reports. Requests for access to these must be addressed to EMA

\section{Ethics approval and consent to participate}

According to Norwegian regulations informed consent and approval from ethics committee are not needed for this report.

\section{Consent for publication}

No information that could allow identification of patients are included. Consent to publish from patients are therefore not necessary.

\section{Competing interests}

THolmøy has received unrestricted research grants to his institution and/or scientific advisory board or speakers honoraria from Biogen, Sanofi, Merck, Novartis, Roche, Santen and Teva; and has participated in clinical trials organized by Biogen, Merck and Roche. L Bø has received unrestricted research grants to his institution and/or scientific advisory board or speakers honoraria from Biogen, Genzyme, Merck, Novartis, Roche and Teva; and has participated in clinical trials organized by Biogen, Merck, Novartis and Roche, and has served as expert witness in litigation for Mylan. B Fevang has received spakers honoraria from MSD and Shire. D Benee Olsen and O Spigset has nothing to disclose.

\section{Author details}

${ }^{1}$ Department of Neurology, Akershus University Hospital, Post Office Box 1000, 1478 Lørenskog, Norway. ${ }^{2}$ Institute of Clinical Medicine, University of Oslo, Oslo, Norway. ${ }^{3}$ Centre for Rare Disorders, Oslo University Hospital, Sognsvannsveien 20, 0372 Oslo, Norway. ${ }^{4}$ Section of Clinical Immunology and Infectious Diseases, Oslo University Hospital, Sognsvannsveien 20, 0372 Oslo, Norway. ${ }^{5}$ Department of Pharmacovigilance, Norwegian Medicines Agency, Post Office Box 240, Skøyen, 0213 Oslo, Norway. ${ }^{6}$ Department of Clinical Pharmacology, St. Olavs University Hospital, Post Office Box 3250, Torgarden, 7006 Trondheim, Norway. ${ }^{7}$ Department of Clinical and Molecular Medicine, Norwegian University of Science and Technology, Trondheim, Norway. ${ }^{8}$ Norwegian Multiple Sclerosis Competence Centre, Department of Neurology, Haukeland University Hospital, Post Office Box 1400, 5021 Bergen, Norway. ${ }^{9}$ Department of Clinical Medicine, University of Bergen, Bergen, Norway.

Received: 8 July 2019 Accepted: 20 July 2019

Published online: 12 August 2019

\section{References}

1. Faissner S, Gold R. Efficacy and safety of the newer multiple sclerosis drugs approved since 2010. CNS Drugs. 2018;32:269-87.

2. Zhang $X$, Tao Y, Chopra M, et al. Differential reconstitution of T cell subsets following immunodepleting treatment with alemtuzumab (anti-CD52 monoclonal antibody) in patients with relapsing-remitting multiple sclerosis. J Immunol. 2013;191:5867-74.

3. European Medical Agency. Lemtrada summary of products characteristics. https://www.ema.europa.eu/ema/index.jsp?curl=pages/medicines/ human/medicines/003718/human_med_001678.jsp\&mid=WC0b01ac05 8001d124. Accessed 19 Mar 2019.

4. Canham LJW, Manara A, Fawcett J, et al. Mortality from Listeria monocytogenes meningoencephalitis following escalation to alemtuzumab therapy for relapsing-remitting Multiple Sclerosis. Mult Scler Relat Disord. 2018;24:38-41.
5. Holmoy T, von der Lippe H, Leegaard TM. Listeria monocytogenes infection associated with alemtuzumab — a case for better preventive strategies. BMC Neurol. 2017;17:65.

6. Myro AZ, Bjerke G, Zarnovicky S, Holmoy T. Diffuse alveolar hemorrhage during alemtuzumab infusion in a patient with multiple sclerosis: a case report. BMC Pharmacol Toxicol. 2018;19:75.

7. Yiannopoulou KG, Papadimitriou D, Anastasiou Al, Siakantaris M. Neutropenia with fatal outcome in a multiple sclerosis patient 23 days after alemtuzumab infusion. Mult Scler Relat Disord. 2018:23:15-6.

8. Metz I, Rieckmann P, Kallmann BA, Bruck W. Disseminated necrotizing leukoencephalopathy eight months after alemtuzumab treatment for multiple sclerosis. Acta Neuropathol Commun. 2016;4:81.

9. Saarela M, Senthil K, Jones J, et al. Hemophagocytic lymphohistiocytosis in 2 patients with multiple sclerosis treated with alemtuzumab. Neurology. 2018;90:849-51.

10. US Food and Drug Administration 2005. Guidance for industry: good pharmacovigilance practices and pharmacoepidemiologic assessment. https://www.fda.gov/files/drugs/published/Good-Pharmacovigilan ce-Practices-and-Pharmacoepidemiologic-Assessment-March-2005.pdf. Accessed 15 May 2019

11. The Uppsala Monitoring Centre. The Use of the WHO-UMC system for standardised casse causality assesment. https://www.who.int/medic ines/areas/quality_safety/safety_efficacy/WHOcausality_assessment.pdf. Accessed 21 Mar 2019.

12. Croteau D, Flowers C, Kulick CG, Brinker A, Kortepeter CM. Acute acalculous cholecystitis: A new safety risk for patients with MS treated with alemtuzumab. Neurology. 2018;90:e1548-52.

13. US Food and Drug Administration 2018. FDA warns about rare but serious risks of stroke and blood vessel wall tears with multiple sclerosis drug Lemtrada (alemtuzumab). Safety Announc. https://www.fda.gov/downl oads/Drugs/DrugSafety/UCM626584.pdf. Accessed 14 Mar 2019.

14. Azevedo CJ, Kutz C, Dix A, Boster A, Sanossian N, Kaplan J. Intracerebral haemorrhage during alemtuzumab administration. Lancet Neurol. 2019;18:329-31.

15. Baker D, Giovannoni G, Schmierer K. Marked neutropenia: Significant but rare in people with multiple sclerosis after alemtuzumab treatment. Mult Scler Relat Disord. 2017;18:181-3.

16. Rieckmann P, Lenz A, Hoffmann M, Poske U, Behr K, Kallmann B. Fatal autoimmune hemolytic anemia associated with alemtuzumab in a MS patient with severe relapsing remitting disease course and prior immune therapies. Neurology. 2016;86:P2.103.

17. Bundesärztekammer. Arzneimittelkommission der Deutschen Ârzteschaft. Theraprefraktäre Autoimmunthrombozytopenie nach Alemtuzumab zur Behandlung einer Multiplen Sklerose. Deutsche Arzteblatt 2017; 114:A2175-6.

18. Baker D, Herrod SS, Alvarez-Gonzalez C, Giovannoni G, Schmierer K. Interpreting lymphocyte reconstitution data from the pivotal phase 3 trials of alemtuzumab. JAMA Neurol. 2017;74:961-9.

19. Thomas K, Eisele J, Rodriguez-Leal FA, Hainke U, Ziemssen T. Acute effects of alemtuzumab infusion in patients with active relapsing-remitting MS Neurol Neuroimmunol Neuroinflam. 2016;3:e228.

20. Wing MG, Moreau T, Greenwood J, et al. Mechanism of first-dose cytokine-release syndrome by CAMPATH 1-H: involvement of CD16 (FcgammaRIII) and CD11a/CD18 (LFA-1) on NK cells. J Clin Invest. 1996:98:2819-26.

21. Cuker A, Bass AD, Nadj C, et al. Immune thrombocytopenia in alemtuzumab-treated MS patients: incidence, detection, and management. Mult Scler. 2019. https://doi.org/10.1177/1352458518816612.

22. Goldman SA. Limitations and strengths of spontaneous reports data. Clin Therap. 1998;20(Suppl C):C40-4.

23. Coles A, Robertson N, Al-Araji A et al. Association of British neurologist 2017. Guidance on the prevention of Listeria infection after alemtuzumab treatment of multiple sclerosis. https://www.theabn.org/news/new-guide lines-on-the-prevention-of-listeria-infection.html. Accessed 15 May 2019.

\section{Publisher's Note}

Springer Nature remains neutral with regard to jurisdictional claims in published maps and institutional affiliations. 